\title{
GPNMB silencing suppresses the proliferation and metastasis of osteosarcoma cells by blocking the PI3K/Akt/mTOR signaling pathway
}

\author{
RUI JIN ${ }^{1}$, YING-YING JIN ${ }^{2}$, YI-LUN TANG ${ }^{3}$, HUA-JUAN YANG ${ }^{1}$, XIAO-QIAN ZHOU ${ }^{1}$ and ZHE LEI ${ }^{1}$ \\ Departments of ${ }^{1}$ Medical Imaging, ${ }^{2}$ Oncology and ${ }^{3}$ Orthopedics, \\ The Second Affiliated Hospital of Xi'an Jiaotong University, Xi'an, Shaanxi 710004, P.R. China
}

Received September 25, 2017; Accepted March 8, 2018

DOI: $10.3892 / o r .2018 .6346$

\begin{abstract}
Glycoprotein non-metastatic melanoma protein B (GPNMB) is a glycoprotein that is highly expressed in various types of cancer, including osteosarcoma. However, its cellular functions and related mechanisms in osteosarcoma remain unclear. In the present study, a higher GPNMB mRNA level was observed in osteosarcoma tissues, than in adjacent non-cancerous tissues. In addition, upregulation of the GPNMB mRNA and protein level was detected in the osteosarcoma cells SaOS2, 143B, MG63 and U2OS using western blot analysis and qPCR. Following transfection with GPNMB siRNA, the proliferation, migration and invasion of MG63 and U2OS cells were assessed using MTT and Transwell assays. The knockdown of GPNMB markedly inhibited the proliferation and metastasis of MG63 and U2OS cells. GPNMB silencing inhibited the activation of PI3K/Akt $/ \mathrm{mTOR}$ signaling in MG63 and U2OS cells. PI3K/AKT activator insulin-like growth factor-1 (IGF-1) significantly activated the $\mathrm{PI} 3 \mathrm{~K} / \mathrm{Akt} / \mathrm{mTOR}$ signaling and reversed the suppressive effects of GPNMB silencing. IGF-1 counteracted the inhibitory effects of GPNMB silencing on the proliferation and metastasis of the MG63 and U2OS cells. In conclusion, we provided evidence that GPNMB silencing regulated the proliferation and metastasis of osteosarcoma cells by suppressing the PI3K/Akt/mTOR signaling pathway. Thus, GPNMB may be a potential therapeutic target for osteosarcoma treatment.
\end{abstract}

\section{Introduction}

Osteosarcoma is the most common malignant bone tumor affecting bone growth, particularly in children and

Correspondence to: Dr Rui Jin, Department of Medical Imaging, The Second Affiliated Hospital of Xi'an Jiaotong University, 157 West Fifth Road, Xincheng, Xi'an, Shaanxi 710004, P.R. China E-mail: racel_327@sina.com

Key words: osteosarcoma, glycoprotein non-metastatic melanoma B, proliferation, migration, invasion, $\mathrm{PI} 3 \mathrm{~K} / \mathrm{Akt} / \mathrm{mTOR}$ adolescents (1). Osteosarcoma is closely associated with lung metastasis, causing death in $\sim 15-25 \%$ of osteosarcoma patients (2). The survival rate for patients with metastatic disease or with tumor recurrence is $<20 \%$ (3). Despite developments in osteosarcoma therapy, the relative 5-year survival rate of osteosarcoma patients remains only $60-70 \%$ due to high malignancy, invasion and metastasis (4). Therefore, it is of great importance to clarify the mechanism of osteosarcoma and develop new therapeutic strategies to prevent metastasis in osteosarcoma and improve patient prognosis.

Glycoprotein non-metastatic melanoma protein B (GPNMB), also known as osteoactivin, encodes the type I transmembrane proteins of 572 amino acids (5). GPNMB contains an extracellular domain, a transmembrane region and a cytoplasmic domain (6,7). GPNMB is expressed in numerous normal tissues, such as bone, the hematopoietic system and skin and it correlates with many biological processes, such as tissue regeneration, inflammation, cell proliferation, adhesion and migration (8). Several studies have reported that GPNMB is also expressed in malignant tissues and influences the metastasis of tumor cells (6,9-11). Furthermore, GPNMB level is reportedly elevated in several malignant cancers, such as uveal melanoma (12), prostate (13) and lung cancer (14). Halim et al (15) reported that GPNMB overexpression is prevalent in osteosarcoma. However, the potential impact of GPNMB on the progression of osteosarcoma remains unclear.

The phosphatidylinositol 3-kinase (PI3K)/Akt/mammalian target of rapamycin (mTOR) signaling pathway is a major signaling cascade. The PI3K/Akt/mammalian target of the mTOR signaling pathway regulates downstream of the receptor tyrosine kinases, including insulin-like growth factor-1 receptor (IGF-1R) (16). This pathway plays a pivotal role in a variety of biological activities that regulate cell growth, survival and migration (17). In addition, researchers have confirmed that abnormalities in the PI3K/Akt/mTOR signaling pathway are involved in the carcinogenesis of various cancers, including osteosarcoma $(18,19)$. This pathway is reportedly activated in osteosarcoma and its suppression could inhibit the proliferation and invasion of osteosarcoma cells (19). Ono et al (20) demonstrated that the extracellular fragment of GPNMB has neuroprotective effects and activates PI3K/Akt pathway. However, whether the PI3K/Akt/mTOR 
pathway is involved in the effects of GPNMB on osteosarcoma remains unclear. The present study was conducted to clarify the role of GPNMB in osteosarcoma. To understand the possible mechanisms involved, the effect of GPNMB on the $\mathrm{PI} 3 \mathrm{~K} / \mathrm{Akt} / \mathrm{mTOR}$ signaling pathway was explored. The results of the present study provided a prospective therapeutic target for osteosarcoma.

\section{Materials and methods}

Cell lines and clinical specimens. The normal human fetal osteoplastic cell line (hFOB) and human osteosarcoma cell lines SaOS2, 143B, MG63 and U2OS were obtained from the American Type Culture Collection (ATCC; Manassas, VA, USA). The cells were cultured in Dulbecco's modified Eagle's medium (Invitrogen; Thermo Fisher Scientific, Inc., Waltham, MA, USA) supplemented with $10 \%$ fetal bovine serum (FBS; Gibco; Thermo Fisher Scientific) at $37^{\circ} \mathrm{C}$ in a humidified $5 \%$ $\mathrm{CO}_{2}$ atmosphere. Twenty paired osteosarcoma and adjacent normal tissues were received from patients in the Second Affiliated Hospital of Xi'an Jiaotong University from January to March, 2017 (Xi'an, China). All patients provided informed written consent and the study was approved by the Institutional Research Ethics Committee of Xi'an Jiaotong University.

Cell transfection. MG63 and U2OS cells were seeded into 6 -well plates $\left(1 \times 10^{5}\right.$ cells/well) and grown for $24 \mathrm{~h}$. Subsequently, MG63 and U2OS cells were transfected with $50 \mu \mathrm{M}$ of GPNMB siRNAs, siGPNMB-1 and siGPNMB-2 or negative control siRNAs, siNC-1 and siNC-2 (all from Thermo Fisher Scientific, Inc.) for $48 \mathrm{~h}$ using Lipofectamine 2000 (Invitrogen; Thermo Fisher Scientific). The transfection efficiency was detected using qPCR and western blot analysis.

MTT assay. The effect of GPNMB on cell viability was assessed by an MTT assay, as previously reported (21). The cells were seeded into 96-well plates at $1 \times 10^{4}$ cells/well. Subsequently, $20 \mu 1$ of MTT (5 mg/ml; Sigma-Aldrich; Merck KGaA, Darmstadt, Germany) was added at 24, 48 and $72 \mathrm{~h}$ and incubated for $4 \mathrm{~h}$. The supernatant was aspirated. Dimethyl sulfoxide (DMSO; $200 \mu \mathrm{l}$; Beijing Dingguo Changsheng Biotechnology Co., Ltd., Beijing, China) was added to dissolve formazan crystals. Absorbance at $490 \mathrm{~nm}$ was detected using a microplate reader (Bio-Tek Instruments, Inc., Winooski, VT, USA).

5'-Bromo-2'-deoxyuridine assay. A 5'-bromo-2'-deoxyuridine (BrdU) assay was performed to assess the effect of GPNMB on cell proliferation, as previously described (22). Cells $\left(1 \times 10^{5}\right.$ cells $\left./ \mathrm{ml}\right)$ grown on coverslips were incubated with BrdU (Sigma-Aldrich; Merck KGaA) for $40 \mathrm{~min}$ and stained with anti-BrdU antibody (1:200; cat. no. sc-70443; Santa Cruz Biotechnology, Santa Cruz, CA, USA) for $2 \mathrm{~h}$. Images were captured under an MC 100 microscope (Carl Zeiss, Oberkochen, Germany), assessing the percentages of BrdU in six random fields.

Transwellassay. To prevent proliferation, the cells were cultured in $10 \mathrm{~g} / \mathrm{ml}$ of mitomycin C (Sigma-Aldrich; Merck KGaA) for $2 \mathrm{~h}$ (23). Then, a Transwell invasion assay was performed using an invasion chamber coated with Matrigel (BD Biosciences,
San Jose, CA, USA). The lower chamber was filled with $600 \mu 1$ of medium. Cells $\left(1 \times 10^{5}\right.$ cells/well $)$ were plated into the upper Transwell chambers in serum-free medium for $24 \mathrm{~h}$ at $37^{\circ} \mathrm{C}$. The cells on the surface of the upper chamber were wiped with a cotton swab. After fixing with methanol for $20 \mathrm{~min}$ and staining with $0.1 \%$ crystal violet for $30 \mathrm{~min}$, the invasive cells on the surface of the bottom membrane were determined by counting five random 100X fields under an MC 100 microscope (Carl Zeiss). The migration assay was the same as the invasion assay, with the exception of the upper chamber which was not coated with Matrigel. Each experiment was performed in triplicate.

Quantitative real-time PCR ( $q P C R)$. Total RNA was extracted using TRIzol reagent (Invitrogen; Thermo Fisher Scientific, Inc.). The synthesis of cDNA was performed with the PrimeScript ${ }^{\mathrm{TM}}$ RT reagent kit (Takara Biotechnology, Co., Ltd., Dalian, China). The qPCR was carried out using SYBR-Green Premix (Takara Biotechnology, Co., Ltd.). The Bio-Rad CFX96 Touch qPCR system (Bio-Rad Laboratories, Hercules, CA, USA) was used to analyze the signal. The primers for qRT-PCR were as follows: GPNMB forward, 5'-ACAAGGAATACA ACCCAATA-3' and reverse, 5'-ATA GCCACTCCAGCACA-3'; GAPDH forward, 5'-GACTCA TGACCACAGTCCATGC-3' and reverse, 5'-AGAGGC AGGGATGATGTTCTG-3'. The expression of mRNAs was normalized to GAPDH. The relative expression was calculated using the $2^{-\Delta \Delta \mathrm{Ct}}$ method.

Western blot analysis. Proteins were resolved in $10 \%$ sodium dodecyl sulfate-polyacrylamide gel electrophoresis (SDS-PAGE), and then the separated proteins were transferred to polyvinylidene fluoride membranes (Invitrogen; Thermo Fisher Scientific, Inc.). Subsequently, the membranes were blocked in $5 \%$ bovine serum albumin (BSA) for $2 \mathrm{~h}$ and probed with primary antibodies, including rabbit anti-GPNMB $(1: 1,000$; cat. no. ab98856), rabbit anti-p-Akt (1:1,000; ab38449), rabbit anti-Akt (1:500; cat. no. ab8805), rabbit anti-p-mTOR (1:1,000; cat. no. ab109268) and rabbit anti-mTOR (1:2,000; cat. no. ab2732; all from Abcam, Cambridge, MA, USA) overnight at $4^{\circ} \mathrm{C}$. Subsequently, the membranes were incubated with horseradish peroxidase-conjugated goat anti-rabbit secondary antibody (1:5,000; cat. no. ab6721; Abcam). The immunoblots were visualized with an enhanced chemiluminescence system (Pierce Biotechnology; Thermo Fisher Scientific, Inc.). Densitometry analysis was performed using Image-Pro Plus 6.0 software (Media Cybernetics Inc., Rockville, MD, USA).

Statistical analysis. The data were analyzed using SPSS 22.0 software (IBM Corp., Armonk, NY, USA) and are presented as the mean \pm SD. Statistical analysis was performed using one-way ANOVA with subsequent SNK-q testing for multiple comparisons. $\mathrm{P}<0.05$ was considered to indicate a statistically significant difference.

\section{Results}

GPNMB expression is upregulated in osteosarcoma. The expression of GPNMB in human osteosarcoma tissues and 
A

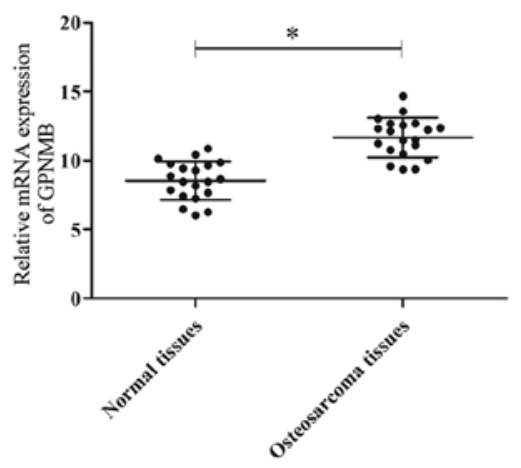

D

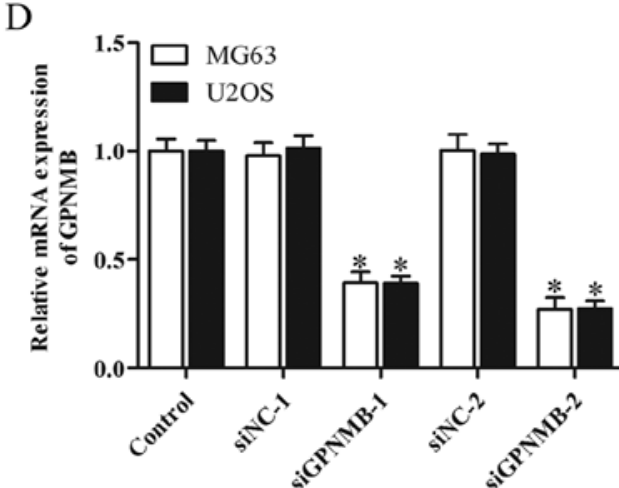

B

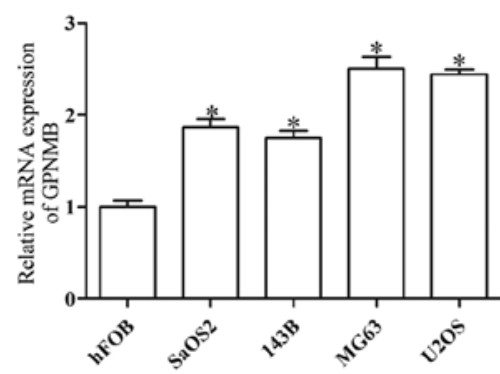

C

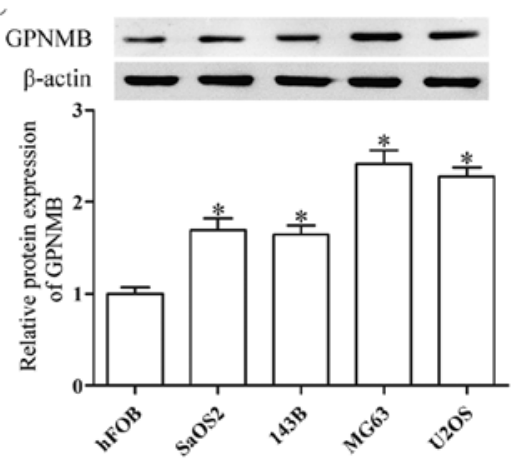

E

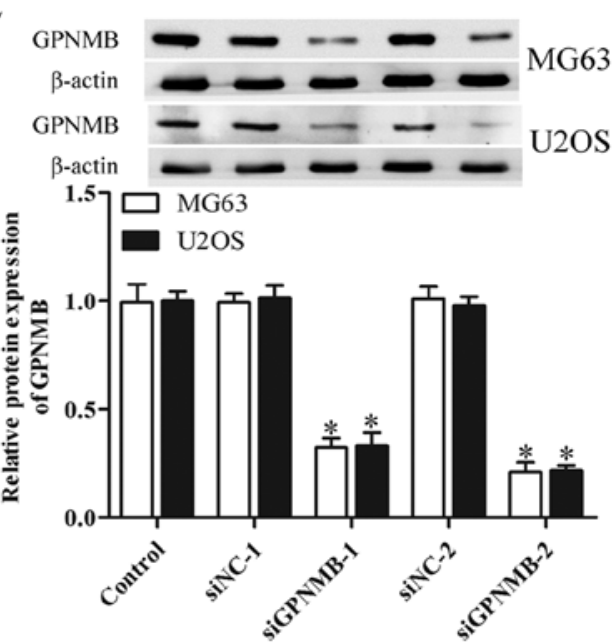

Figure 1. GPNMB is overexpressed in human osteosarcoma tissues and human osteosarcoma cell lines. (A) The mRNA expression of GPNMB in human osteosarcoma tissues and non-cancerous tissues was detected by qPCR. N=20, ${ }^{*} \mathrm{P}<0.05$ vs. adjacent non-cancerous tissues. (B) The mRNA and (C) protein levels of GPNMB in human osteosarcoma cell lines SaOS2, 143B, MG63 and U2OS were detected by qPCR and western blot analysis. N=3, * $<0.05$ vs. hFOB. (D and E) Interference efficiency was determined by qPCR and western blot analysis. siGPNMB-1 and siGPNMB-2 significantly downregulated the (D) mRNA and (E) protein expression of GPNMB in MG63 and U2OS cells. $\mathrm{N}=3,{ }^{*} \mathrm{P}<0.05$ vs. the control. hFOB, human fetal osteoplastic cells.

human osteosarcoma cell lines was determined by qPCR and western blot analysis. The human osteosarcoma tissues derived from 20 patients demonstrated significantly higher GPNMB mRNA levels than the adjacent non-cancerous tissues (Fig. 1A). The mRNA and protein expression of GPNMB in the osteosarcoma cell lines SaOS2, 143B, MG63 and U2OS was upregulated in comparison with normal human fetal osteoplastic cells (hFOBs) (Fig. 1B and C). The MG63 and $\mathrm{U} 2 \mathrm{OS}$ cells indicated higher GPNMB mRNA and protein expression than the SaOS2 and 143B cells. Therefore, these two cell lines were chosen for further study.

To investigate the functions of GPNMB in relation to osteosarcoma, we separately silenced its expression in the MG63 and U2OS cells via GPNMB siRNA transfection. A high inhibitory GPNMB mRNA and protein level was found in the MG63 and U2OS cells transfected with GPNMB siRNA (P<0.05; Fig. 1D and E). Of the two GPNMB siRNAs (siGPNMB-1 and siGPNMB-2), siGPNMB-2 was the most efficient and thus was chosen for further experiments.

GPNMB silencing inhibits proliferation and metastasis of osteosarcoma cells. Knockdown of GPNMB in the MG63 and U2OS cells resulted in reduced cell proliferation (Fig. 2A and B). The Transwell assay indicated that GPNMB silencing notably inhibited the migration (Fig. 2C and D) and invasion (Fig. 2E and F) of the MG63 and U2OS cells. These results indicated that GPNMB may promote the progression of osteosarcoma.

GPNMB silencing suppresses the activity of the PI3K/Akt/mTOR signaling pathway in osteosarcoma cells. The abnormal activation of the PI3K/Akt/mTOR signaling pathway plays a critical role in osteosarcoma pathogenesis $(24,25)$. Therefore, we further evaluated whether GPNMB affected the PI3K/Akt/mTOR signaling pathway in osteosarcoma cells. The protein levels of p-PI3K, PI3K, p-AKT, AKT, p-mTOR and mTOR were obviously upregulated in the MG63 and U2OS cells (Fig. 3A), whereas the protein levels were significantly downregulated by GPNMB siRNA in both MG63 (Fig. 3B) and U2OS cells (Fig. 3C). These results indicated that the GPNMB silencing inhibited the activation of the PI3K/Akt/mTOR signaling pathway in osteosarcoma cells.

GPNMB silencing suppresses the proliferation and metastasis of osteosarcoma cells by blocking the PI3K/Akt/mTOR 
A

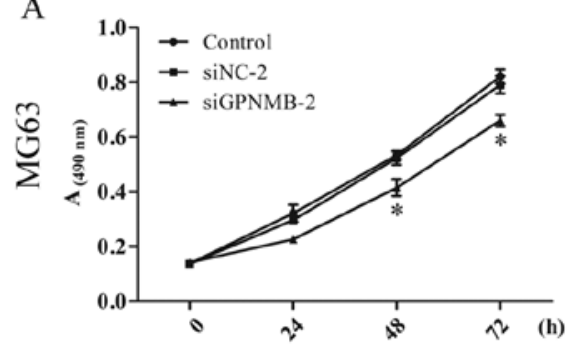

B

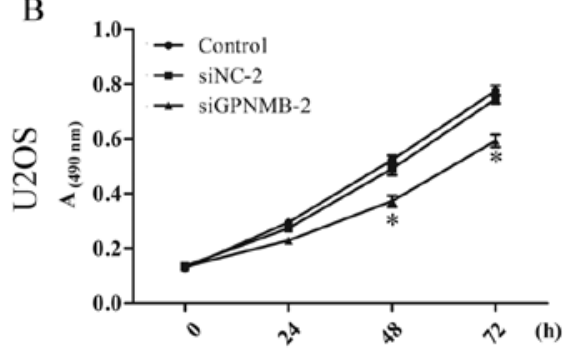

C

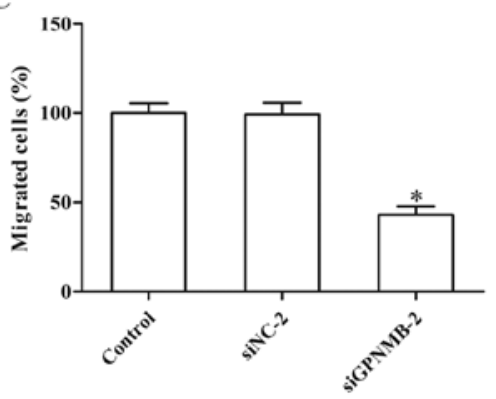

D

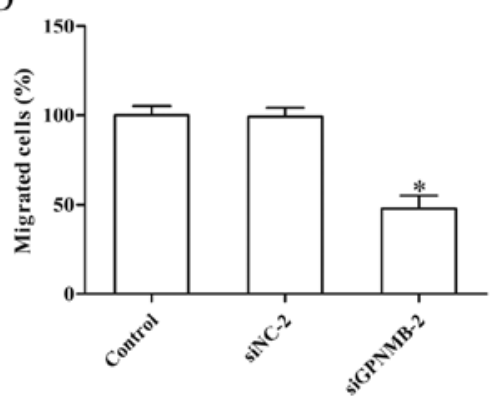

E

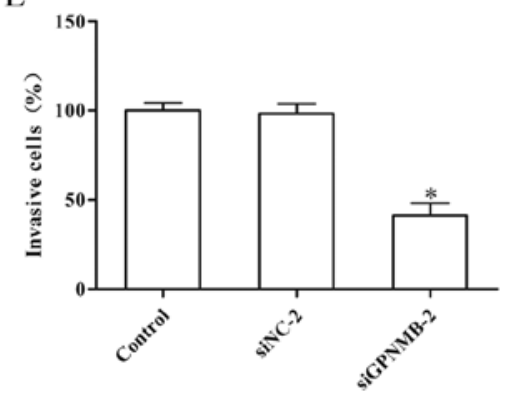

$\mathrm{F}$

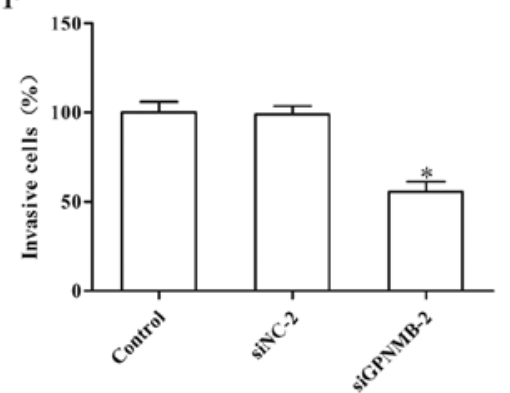

Figure 2. GPNMB silencing inhibits the proliferation, migration and invasion of osteosarcoma cells. The proliferation of (A) MG63 and (B) U2OS cells was detected by an MTT assay on three consecutive days. The siGPNMB-2-transfected cells led to a significant decrease in the proliferation of MG63 and U2OS cells. (C and E) Cell migration and invasion of MG63 and (D and F) U2OS cells were detected by Transwell assay. siGPNMB-2 significantly inhibited cell migration and invasion in MG63 and $\mathrm{U} 2 \mathrm{OS}$ cells. $\mathrm{N}=3,{ }^{*} \mathrm{P}<0.05$ vs. the control.

A
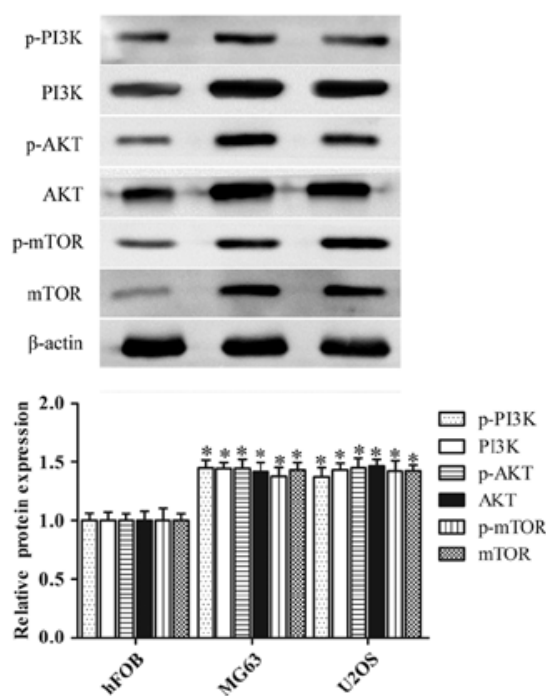

B
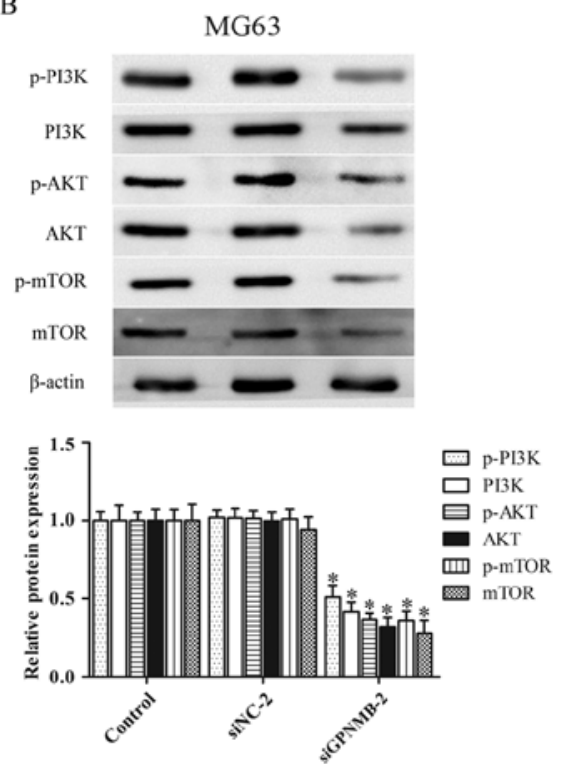

$\mathrm{C}$
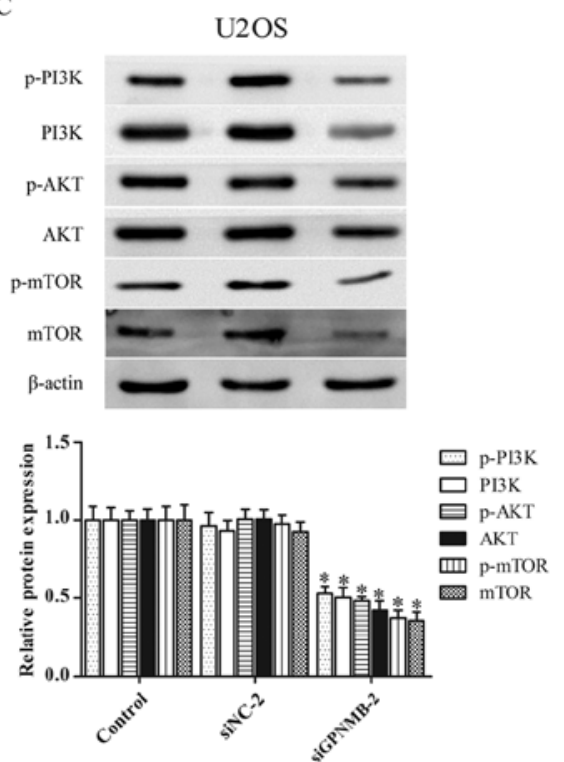

Figure 3. GPNMB silencing suppresses the activation of the PI3K/Akt/mTOR signaling pathway in osteosarcoma cells. (A) The protein expression of p-PI3K, PI3K, p-AKT, AKT, p-mTOR and mTOR was upregulated in MG63 and U2OS cells. N=3, ${ }^{*} \mathrm{P}<0.05$ vs. hFOB cells. (B and C) GPNMB silencing inhibited the $\mathrm{PI} 3 \mathrm{~K} / \mathrm{Akt} / \mathrm{mTOR}$ signaling pathway-related proteins in (B) MG63 and (C) $\mathrm{U} 2 \mathrm{OS}$ cells. $\mathrm{N}=3,{ }^{\mathrm{P}} \mathrm{P}<0.05$ vs. control.

signaling pathway. To identify whether the effect of GPNMB silencing on osteosarcoma cells was achieved by regulating the PI3K/Akt/mTOR signaling pathway, the MG63 and U2OS cells were cultured with IGF-1 (3 ng/ml) (26), an agonist of PI3K. IGF-1 activated the PI3K/Akt/mTOR signaling and abolished the inhibition of PI3K/Akt/mTOR signaling induced by siGPNMB-2, as evidenced by the upregulation of the protein expression of p-PI3K, PI3K, p-AKT, AKT, p-mTOR and mTOR (Fig. 4A and B).

Furthermore, the inhibitory effect of GPNMB silencing on the proliferation (Fig. 4C and F), migration (Fig. 4D and G) and invasion (Fig. 4E and $\mathrm{H}$ ) of the MG63 and U2OS cells was significantly reversed by IGF-1. These results demonstrated that GPNMB silencing inhibited the progression of 
A
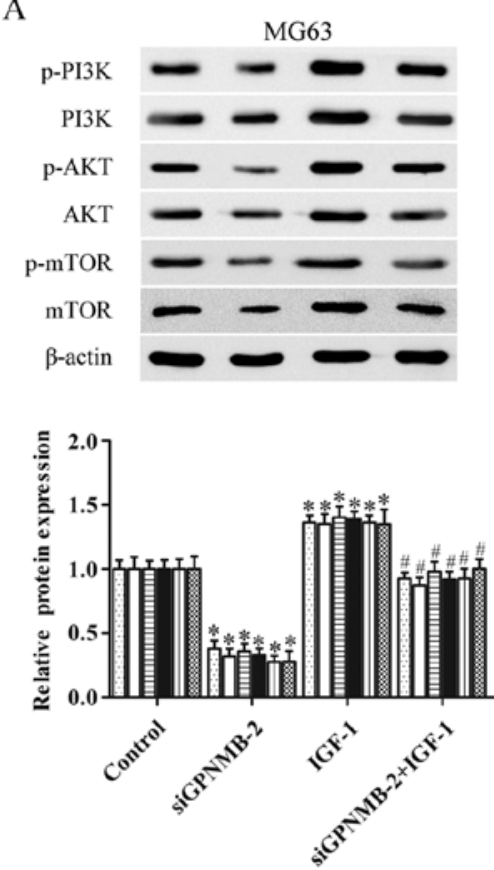

B

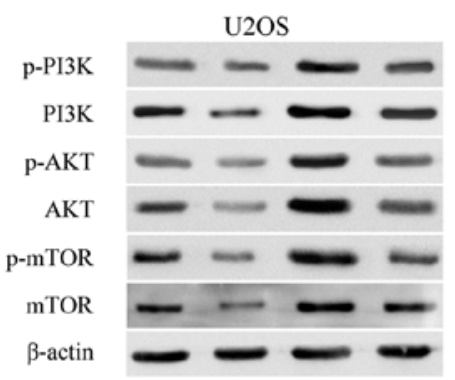

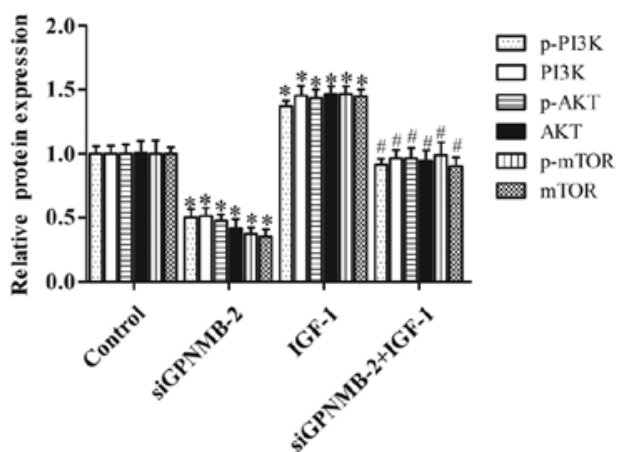

C

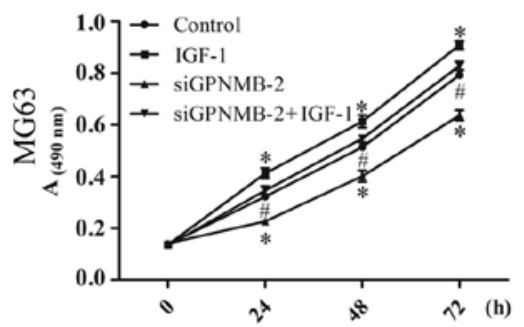

D

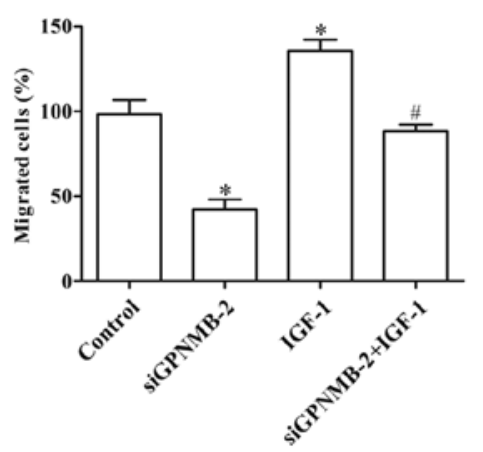

G

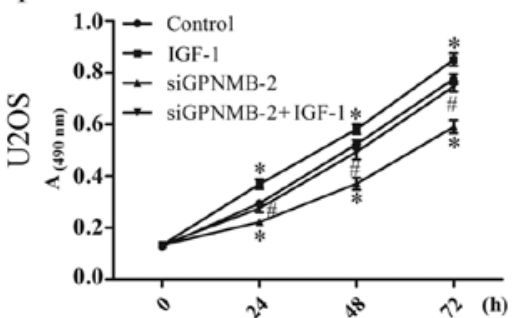

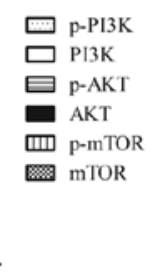

E

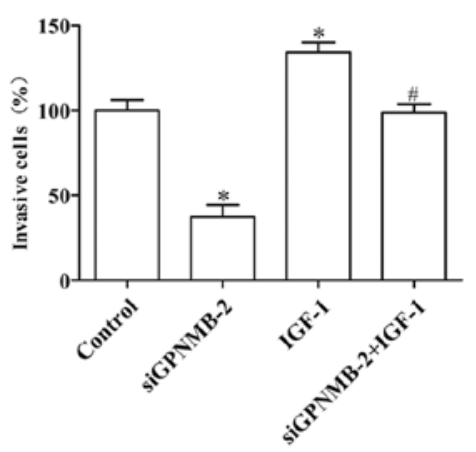

$\mathrm{H}$

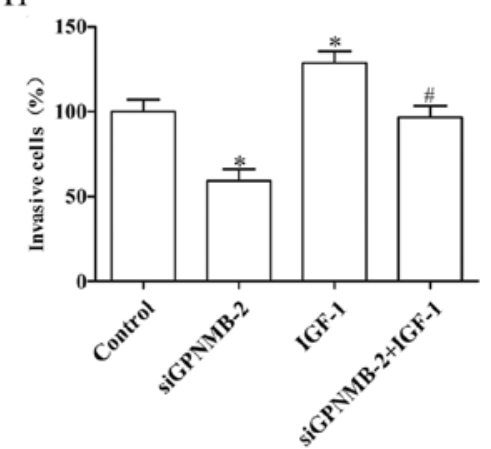

Figure 4. GPNMB silencing inhibits osteosarcoma tumorigenesis by suppressing the PI3K/Akt/mTOR pathway. The cells were treated with IGF-1 (3 ng/ml) to stimulate the PI3K/Akt/mTOR signaling pathway. (C-H) IGF-1 efficiently reversed the inhibitory effects of GPNMB siRNA on the PI3K/Akt/mTOR pathway in (A) MG63 and (B) U2OS cells. IGF-1 counteracted the effects of siGPNMB-2 on the (C and F) proliferation, (D and G) migration and (E and H) invasion of the MG63 and U2OS cells. N=3, ${ }^{\mathrm{P}}<0.05$ vs. the control; ${ }^{*} \mathrm{P}<0.05$ vs. siGPNMB-2.

osteosarcoma cells by blocking the PI3K/Akt/mTOR signaling pathway.

\section{Discussion}

Osteosarcoma is the most common bone tumor with a high mortality rate. In the present study, we revealed that glycoprotein non-metastatic melanoma protein B (GPNMB) was aberrantly overexpressed in osteosarcoma tissue and osteosarcoma cells. The knockdown of the expression of GPNMB significantly inhibited the progression of osteosarcoma cells by inhibiting the PI3K/Akt/mTOR signaling pathway. Our findings indicated that GPNMB may be a promising therapeutic target for osteosarcoma. GPNMB is 
a transmembrane glycoprotein involved in various pathological processes, including the development of cancer. It has been reported to increase cellular survival signals, thus promoting tumor growth (6). In addition, GPNMB is highly expressed in various types of cancer, including osteosarcoma (15). The results from a pediatric preclinical testing program of solid tumor xenografts revealed that GPNMB was primarily expressed in osteosarcoma xenografts (27). In a clinical trial, targeting GPNMB with the antibody-drug conjugate glembatumumab vedotin demonstrated the potential utility of targeting GPNMB for the treatment of osteosarcoma (28), however, its role in human osteosarcoma remains unclear.

In the present study, the expression of GPNMB was highly expressed in osteosarcoma tissue and osteosarcoma cells. To elucidate the biological function of GPNMB in osteosarcoma cells, we used GPNMB siRNA to knock down the expression of GPNMB. GPNMB silencing inhibited the proliferation, migration and invasion of the MG63 and U2OS cells. Collectively, these results indicated that GPNMB plays a vital role in the tumorigenicity and progression of osteosarcoma, which is consistent with the role of GPNMB in other cancers $(14,29,30)$.

The PI3K/Akt/mTOR signaling pathway plays a critical role in many biological processes, such as the survival, migration and progression of various types of cancer $(31,32)$. PI3K activates its downstream molecule Akt, the core component of the PI3K/AKT signaling pathway, which in turn results in the phosphorylation and activation of mTOR through a cascade of regulators and ultimately increases the proliferation and metastasis of the tumor cells (33). A previous study has revealed that the aberrant activation of the $\mathrm{PI} 3 \mathrm{~K} / \mathrm{Akt} / \mathrm{mTOR}$ signaling pathway was a pivotal event in the pathogenesis and progression of osteosarcoma cells (34). To investigate the mechanisms of GPNMB silencing in the progression of osteosarcoma cells, we assessed the effect of GPNMB silencing on the PI3K/Akt/mTOR signaling pathway-related proteins p-PI3K, PI3K, p-AKT, AKT, p-mTOR and mTOR. Consistent with previous studies $(35,36)$, we revealed that the PI3K/Akt/mTOR signaling pathway was activated in osteosarcoma cells, however the GPNMB silencing inhibited the activation of the PI3K/Akt/mTOR signaling pathway. The present study also revealed that the inhibitory effect of GPNMB silencing on the proliferation, migration and invasion of the MG63 and U2OS cells was partly reversed by the PI3K/Akt agonist IGF-1. These results demonstrated that the GPNMB silencing exerted an inhibitory effect on the progression of osteosarcoma by suppressing the PI3K/Akt/mTOR signaling pathway. However, IGF-1 did not completely reverse the effect of GPNMB, indicating that other signaling pathways may be involved in the effect of GPNMB on osteosarcoma. Future research is warranted to further investigate these pathways.

In conclusion, the present study revealed that GPNMB was highly expressed in osteosarcoma and that GPNMB silencing inhibited the proliferation and metastasis of osteosarcoma cells by suppressing the PI3K/Akt/mTOR signaling pathway in vitro. Other possible effects of GPNMB should be investigated in vivo, as GPNMB may be a potential therapeutic target for the treatment of osteosarcoma.

\section{Acknowledgements}

Not applicable.

\section{Funding}

No funding was received.

\section{Availability of data and materials}

The osteosarcoma and adjacent normal tissues were received from patients in the Second Affiliated Hospital of Xi'an Jiaotong University from January to March, 2017 (Xi'an, China).

\section{Authors' contributions}

RJ and YYJ conceived and designed the study. RJ, YYJ, YLT and HJY performed the experiments. YLT and ZL analyzed the data. RJ and XQZ wrote and reviewed the manuscript. All authors read and approved the manuscript and agree to be accountable for all aspects of the research in ensuring that the accuracy or integrity of any part of the work are appropriately investigated and resolved.

\section{Ethics approval and consent to participate}

All patients provided informed written consent and the study was approved by the Institutional Research Ethics Committee of Xi'an Jiaotong University.

\section{Consent for publication}

Not applicable.

\section{Competing interests}

The authors declare that they have no competing interests.

\section{References}

1. Vasquez L, Tarrillo F, Oscanoa M, Maza I, Geronimo J, Paredes G, Silva JM and Sialer L: Analysis of prognostic factors in high-grade osteosarcoma of the extremities in children: A 15-year single-institution experience. Front Oncol 6: 22, 2016.

2. Gordon $\mathrm{N}$ and Kleinerman ES: Aerosol therapy for the treatment of osteosarcoma lung metastases: Targeting the Fas/FasL pathway and rationale for the use of gemcitabine. J Aerosol Med Pulm Drug Deliv 23: 189-196, 2010.

3. Ferguson WS and Goorin AM: Current treatment of osteosarcoma. Cancer Invest 19: 292-315, 2001.

4. Broadhead ML, Clark JC, Myers DE, Dass CR and Choong PF: The molecular pathogenesis of osteosarcoma: A review. Sarcoma 2011: 959248, 2011.

5. Safadi FF, Xu J, Smock SL, Rico MC, Owen TA and Popoff SN: Cloning and characterization of osteoactivin, a novel cDNA expressed in osteoblasts. J Cell Biochem 84: 12-26, 2001.

6. Maric G, Rose AA, Annis MG and Siegel PM: Glycoprotein non-metastatic b (GPNMB): A metastatic mediator and emerging therapeutic target in cancer. Onco Targets Ther 6: 839-852, 2013.

7. Rose AA, Annis MG, Dong Z, Pepin F, Hallett M, Park M and Siegel PM: ADAM10 releases a soluble form of the GPNMB/Osteoactivin extracellular domain with angiogenic properties. PLoS One 5: e12093, 2010.

8. Singh M, Del Carpio-Cano F, Belcher JY, Crawford K, Frara N, Owen TA, Popoff SN and Safadi FF: Functional roles of osteoactivin in normal and disease processes. Crit Rev Eukaryot Gene Expr 20: 341-357, 2010. 
9. Rose AA and Siegel PM: Osteoactivin/HGFIN: Is it a tumor suppressor or mediator of metastasis in breast cancer? Breast Cancer Res 9: 403, 2007.

10. Maric G, Annis MG, Dong Z, Rose AA, Ng S, Perkins D, MacDonald PA, Ouellet V, Russo C and Siegel PM: GPNMB cooperates with neuropilin-1 to promote mammary tumor growth and engages integrin $\alpha 5 \beta 1$ for efficient breast cancer metastasis. Oncogene 34: 5494-5504, 2015.

11. Zhuo H and Zhou L: Gpnmb/osteoactivin: An indicator and therapeutic target in tumor and nontumorous lesions. Pharmazie 71: 555-561, 2016.

12. Williams MD, Esmaeli B, Soheili A, Simantov R, Gombos DS, Bedikian AY and Hwu P: GPNMB expression in uveal melanoma: A potential for targeted therapy. Melanoma Res 20: 184-190, 2010

13. Fiorentini C, Bodei S, Bedussi F, Fragni M, Bonini SA Simeone C, Zani D, Berruti A, Missale C, Memo M, et al: GPNMB/OA protein increases the invasiveness of human metastatic prostate cancer cell lines DU145 and PC3 through MMP-2 and MMP-9 activity. Exp Cell Res 323: 100-111, 2014.

14. Oyewumi MO, Manickavasagam D, Novak K, Wehrung D, Paulic N, Moussa FM, Sondag GR and Safadi FF: Osteoactivin (GPNMB) ectodomain protein promotes growth and invasive behavior of human lung cancer cells. Oncotarget 7: 13932-13944, 2016.

15. Halim A, Bagley RG and Keler T: Abstract 5032: Glycoprotein NMB (gpNMB) overexpression is prevalent in human cancers: Pancreatic cancer, non-small cell lung cancer, head and neck cancer, and osteosarcoma. Cancer Res 76: 5032-5032, 2016.

16. Ludwig JA, Lamhamedi-Cherradi SE, Lee HY, Naing A and Benjamin R: Dual targeting of the insulin-like growth factor and collateral pathways in cancer: combating drug resistance. Cancers 3: 3029-3054, 2011.

17. Ke M, Mo L, Li W, Zhang X, Li F and Yu H: Ubiquitin ligase SMURF1 functions as a prognostic marker and promotes growth and metastasis of clear cell renal cell carcinoma. FEBS Open Bio 7: 577-586, 2017.

18. Yuan TL and Cantley LC: PI3K pathway alterations in cancer: Variations on a theme. Oncogene 27: 5497-5510, 2008.

19. Liu Y, Bi T, Dai W, Wang G, Qian L, Shen G and Gao Q: Lupeol induces apoptosis and cell cycle arrest of human osteosarcoma cells through PI3K/AKT/mTOR pathway. Technol Cancer Res Treat 15: NP16-NP24, 2016.

20. Ono Y, Tsuruma K, Takata $M$, Shimazawa $M$ and Hara $H$ : Glycoprotein nonmetastatic melanoma protein B extracellular fragment shows neuroprotective effects and activates the PI3K/Akt and MEK/ERK pathways via the $\mathrm{Na}^{+} / \mathrm{K}^{+}$-ATPase. Sci Rep 6: 23241, 2016.

21. Zhu H, Wu Y, Zheng $\mathrm{W}$ and Lu S: CO-029 is overexpressed in gastric cancer and mediates the effects of EGF on gastric cancer cell proliferation and invasion. Int J Mol Med 35: 798-802, 2015

22. Chen L, Wang Q, Wang GD, Wang HS, Huang Y, Liu XM and Cai XH: miR-16 inhibits cell proliferation by targeting IGF1R and the Raf1-MEK1/2-ERK1/2 pathway in osteosarcoma. FEBS Lett 587: 1366-1372, 2013.

23. Boniface K, Bernard FX, Garcia M, Gurney AL, Lecron JC and Morel F: IL-22 inhibits epidermal differentiation and induces proinflammatory gene expression and migration of human keratinocytes. J Immunol 174: 3695-3702, 2005.
24. Wang Y, Sun Y, Wu Y and Zhang J: Cucurbitacin E inhibits osteosarcoma cells proliferation and invasion through attenuation of PI3K/AKT/mTOR signaling. Biosci Rep: BSR20160165, 2016.

25. Hu K, Dai HB and Qiu ZL: mTOR signaling in osteosarcoma: Oncogenesis and therapeutic aspects (Review). Oncol Rep 36: $1219-1225,2016$

26. Zhang XJ, Yu HY, Cai YJ and Ke M: Lycium barbarum polysaccharides inhibit proliferation and migration of bladder cancer cell lines BIU87 by suppressing Pi3K/AKT pathway. Oncotarget 8: 5936-5942, 2017.

27. Kolb EA, Gorlick R, Billups CA, Hawthorne T, Kurmasheva RT, Houghton PJ and Smith MA: Initial testing (stage 1) of glembatumumab vedotin (CDX-011) by the pediatric preclinical testing program. Pediatr Blood Cancer 61: 1816-1821, 2014.

28. Roth M, Barris DM, Piperdi S, Kuo V, Everts S, Geller D, Houghton P, Kolb EA, Hawthorne T, Gill J and Gorlick R: Targeting glycoprotein NMB with antibody-drug conjugate, glembatumumab vedotin, for the treatment of osteosarcoma. Pediatr Blood Cancer 63: 32-38, 2016.

29. Rose AA, Grosset AA, Dong Z, Russo C, Macdonald PA, Bertos NR, St-Pierre Y, Simantov R, Hallett M, Park M, et al: Glycoprotein nonmetastatic B is an independent prognostic indicator of recurrence and a novel therapeutic target in breast cancer. Clin Cancer Res 16: 2147-2156, 2010.

30. Torres C, Linares A, Alejandre MJ, Palomino-Morales R, Martin M, Delgado JR, Martinez J and Perales S: The potential role of the glycoprotein osteoactivin/glycoprotein nonmetastatic melanoma protein B in pancreatic cancer. Pancreas 44: 302-310, 2015.

31. Le B, Powers GL, Tam YT, Schumacher N, Malinowski RL, Steinke L, Kwon G and Marker PC: Multi-drug loaded micelles delivering chemotherapy and targeted therapies directed against HSP90 and the PI3K/AKT/mTOR pathway in prostate cancer. PloS One 12: e0174658, 2017.

32. Liu M, Bamodu OA, Huang WC, Zucha MA, Lin YK, Wu ATH, Huang CC, Lee WH, Yuan CC, Hsiao M, et al: 4-Acetylantroquinonol B suppresses autophagic flux and improves cisplatin sensitivity in highly aggressive epithelial cancer through the PI3K/Akt/mTOR/p70S6K signaling pathway. Toxicol Appl Pharmacol 325: 48-60, 2017.

33. Rodon J, Dienstmann R, Serra V and Tabernero J: Development of PI3K inhibitors: Lessons learned from early clinical trials. Nat Rev Clin Oncol 10: 143-153, 2013.

34. Li X, Sun X, Wu J and Li Z: MicroRNA-613 suppresses proliferation, migration and invasion of osteosarcoma by targeting c-MET. Am J Cancer Res 6: 2869-2879, 2016.

35. Song R, Tian K, Wang W and Wang L: P53 suppresses cell proliferation, metastasis, and angiogenesis of osteosarcoma through inhibition of the PI3K/AKT/mTOR pathway. Int J Surg 20: 80-87, 2015.

36. Keremu A, Maimaiti X, Aimaiti A, Yushan M, Alike Y, Yilihamu Y and Yusufu A: NRSN2 promotes osteosarcoma cell proliferation and growth through PI3K/Akt/MTOR and Wnt/ $\beta$-catenin signaling. Am J Cancer Res 7: 565-573, 2017. 